\title{
The Cost of Chronic Obstructive Pulmonary Disease (COPD) in Compliant Versus Noncompliant Patients
}

\author{
Kimberly Stephens Rudd ${ }^{1}$ and Tiffany C. Jackman ${ }^{1}$ \\ ${ }^{1}$ University of West Florida, Pensacola, FL, USA \\ DOI: https://doi.org/10.47611/jsr.v10i1.797
}

\begin{abstract}
A literature review conducted on the cost of Chronic Obstructive Pulmonary Disease (COPD) between patients who are compliant versus non-compliant reflects on the data for the United States, emphasizing the areas within the Florida Panhandle. An overview of costs associated with non-compliant COPD patients and strategies for encouraging patients to adhere to treatment and improve healthcare outcomes is provided. A review of recent peer-reviewed articles was performed to determine the additional costs associated with non-compliance. The direct cost of COPD was estimated at $\$ 6,246$ per patient annually. Nationally projected COPD expenditures were $\$ 49.9$ billion in 2010 , while costs in Florida were $\$ 2.5$ billion. Florida's costs for COPD management ranks much higher than the national average. Potential causes for the differentiation are associated with newer medications and devices that contribute to a higher out-ofpocket expense for patients, patient denial about their illness's seriousness, maybe the inability to understand compliance with treatment. The relationship between the primary care provider and COPD patient is critical for adherence to therapy.
\end{abstract}

\section{Research Overview/Question}

A State-of-the-Art literature review was conducted on the cost of Chronic Obstructive Pulmonary Disease (COPD), between patients who are compliant versus non-compliant, by reviewing data for the United States and emphasizing the areas of the Florida Panhandle. The objective of this research is to obtain a better understanding of the following:

1. What is the cost of non-compliance for COPD patients?

2. What are some reasons that patients choose to be non-compliant?

3. What can be done to assist a patient with becoming more compliant with their treatment?

\section{Introduction}

COPD is characterized by the American Thoracic Society (2013) as difficulty breathing on exhalation. This symptomology occurs when airflow is obstructed in the lungs, and exhalation is not completed. An individual exhales a small percentage of the air intake. The remaining air gets trapped in the lungs causing shortness of breath, tiredness, and chest discomfort. An individual with COPD may complain of not able to get a full breath. Although several factors contribute to COPD, the majority of COPD cases are linked to cigarette smoke (American Thoracic Society, 2013). Environmental factors include pollution, dust, and chemicals (American Thoracic Society, 2013). Recent genetics research has provided new insight into COPD development later in life (American Thoracic Society, 2013). 
An overview of costs associated with non-compliant COPD patients and strategies for encouraging patients to adhere to treatment and improve healthcare outcomes is provided throughout the literature. A review of recent peerreviewed articles was performed to determine the additional costs associated with non-compliance. This study also investigates potential causes specific to the Florida panhandle region based on readmission data on COPD patients.

\section{Patient Demographics}

The background of a patient is within the Panhandle and was gathered using the BRFSS for 2011. Within the youngest age group, eighteen to forty-four were four percent diagnosed with COPD (CDC, 2018). The majority of people are diagnosed after the age of forty (CDC, 2018). Women are more likely to have COPD than men by two percent (CDC, 2018). COPD patient's income is typically less than 50,000 a year. Only $14 \%$ of patients with COPD are eligible for Medicare due age (CDC, 2018). Residents that carry the COPD diagnosis stated cost hindered receiving appropriate care (CDC, 2018).

\section{Methods}

The research plan to review the most recent peer-reviewed articles from journals dates between 2016 to present was chosen to determine the additional costs of COPD for non-compliant individuals with a subsequent drill down to the Florida panhandle region. The projected outcome expected that non-compliant individuals accumulated more cost more than compliant individuals. The data was based on the Florida Department of Health data tracker. The data was compiled by outlining the costs associated with non-compliance and potential causes for the differentiation. Peerreviewed journal articles were chosen using the following:

1. Databases: Florida Department of Health (FDOH), Agency for Health Care Administration (AHCA), Centers for Disease Control and Prevention (CDC), PubMed

2. Keywords: Chronic Obstructive Pulmonary Disease, COPD, Florida, Compliance, Healthcare Costs

3. Inclusion/Exclusion: Scholarly Peer Review within the last five years

\section{Results}

COPD's direct cost was estimated at $\$ 6,246$ per patient annually (Patel et al., 2018). Nationally projected COPD expenditures were $\$ 49.9$ billion in 2010, while costs in Florida were $\$ 2.5$ billion (CDC, 2018). According to Restrepo, Alvarez, Wittnebel, Sorenson, Wettstein, Vines, Sikkema-Ortiz, Gardner, \& Wilkins (2008), potential causes for the differentiation are associated with newer medications and devices that contribute to a higher out-of-pocket expense for patients, patient denial about the seriousness of their illness, maybe the inability to understand compliance with treatment.

There are several treatments to treat COPD. Patients are more compliant when using fewer medications than having one of each classification. There are long-acting beta 2 (LABA), a long-acting muscarinic antagonist (LAMA), or inhaled corticosteroid (ICS), and medications that combine the types mentioned above of classifications into one inhaler (Bhattarai et al., 2020). Examples are ICS/LABA or LABA/LAMA or ICS/ LABA/LAMA (Bhattarai et al., 2020). LABA are long-acting bronchodilators that last twelve hours. LAMA works on the parasympathetic nervous system that brochodilator and assists in decreasing muscous and assisting in mucous production. ICS reduces inflammation within the bronchial tubes. Compliant COPD patients with inhaled corticosteroid/LABA treatments had lower COPD exacerbation rates and healthcare costs than non-compliant patients (Davis et al., 2017). Better adherence to 
maintenance therapies can reduce the economic burden of COPD. Thus, the relationship between the primary care provider and COPD patient is critical to therapy (Restrepo et al., 2008). Bhattarai colleagues found a clinical study that patients' satisfaction with physicians increased or decreased their compliance (Bhattarai et al., 2020).

Previous studies found an average additional cost of $\$ 3,000$ to $\$ 4,000$ difference for non-compliant COPD patients, including higher rates for pharmacy, skilled nursing facilities, outpatient services, emergency services, and hospitalization costs (Guarasico et al., 2013; Davis et al., 2017). An estimated 19.4 million days of work were lost nationally due to COPD-related illness (Patel et al., 2018). Between April 2017 and March 2018, Florida averaged 63,572 hospitalizations for COPD, costing between $\$ 23,645$ and $\$ 55,492$, with a 4.3 days average length of stay (AHCA, 2017). The average costs for a care bundle, including two visits with a primary care physician for evaluation, nationally averages $\$ 225$, Florida averages of $\$ 186$, while the Florida Panhandle average cost for COPD soars at \$231 for in-network providers (AHCA, 2017).

Preventing exacerbations is estimated to save $\$ 4$ billion for COPD patients. According to Patel and colleagues (2018), the average costs for non-compliant patients were $\$ 17,651$ year versus $\$ 11,395 /$ year $(\mathrm{P}<0.001)$ for compliant patients. For this literature review, documented emergency department visits for exacerbators were considered noncompliant COPD episodes, further increasing COPD expenditures.

Although the rural counties reported a higher prevalence of COPD (Figure 1), the emergency department admissions were slightly lower, suggesting compliance with treatment regimens or only being less populated (Figure 2).

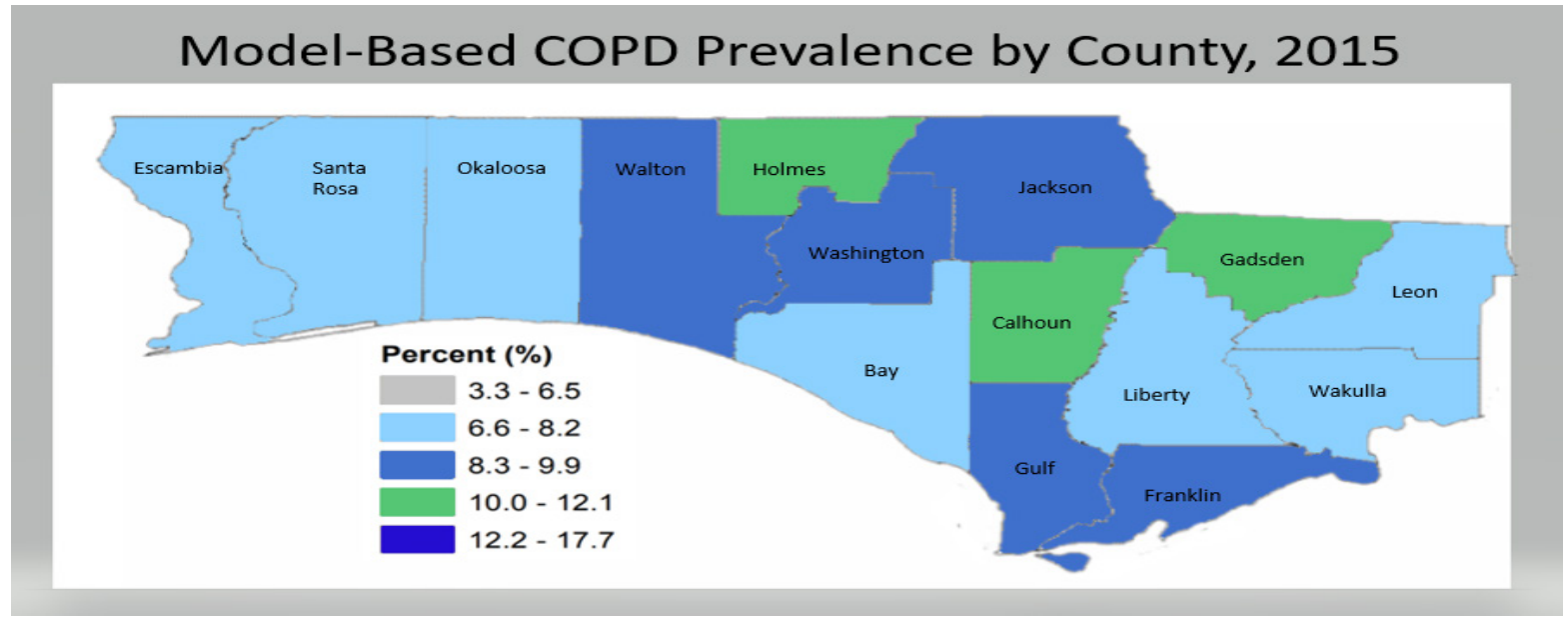

Figure 1. Model-Based COPD Prevalence by County, 2015

(CDC. Urban-Rural County and State Differences in Chronic Obstructive pulmonary Disease-United States, 2015) 


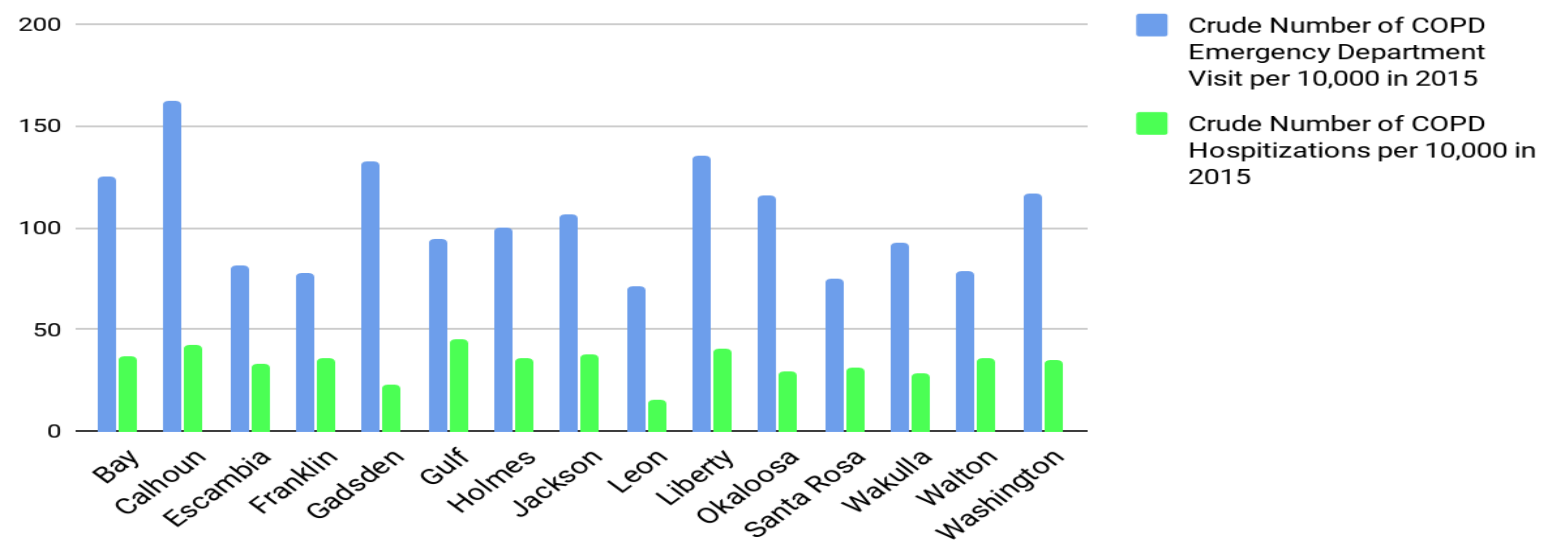

Figure 2. Florida Panhandle County Compare of COPD Hospitalizations \& ED Visits

Although the costs associated with non-compliance was significantly higher, several reasons were cited for COPD patients choosing to be non-compliant with treatment. The patient's understanding of the treatment plan, misuse (overuse, underuse, or improperly used) of therapy medications decreases the ability to determine the effectiveness of therapy and the patient's inability to inhale medication for optimal medication deposition in the lungs (Smith et al., 2017). There are two different behaviors of patients that contribute to non-compliance intentional and unintentional. Intentional non-compliance often results from the patient's inability to understand their disease process and how the medications work (Garvey \& Kaplan, 2018). Unintentional non-compliance results from the patient's COPD being treated with several medications creating a complex therapy for the patient to follow (Garvey \& Kaplan, 2018).

\section{Conclusion}

The relationship between the primary care provider and COPD patient is critical to adherence to therapy. Individuals with COPD should be educated on the seriousness of this disease, how the medication works, why it is essential to be compliant with treatment plans, and education on the proper use of all medications to ensure proper medication delivery for maximal therapy (Sethi, 2018). To achieve this type of care, the desideratum of incorporating respiratory therapists in outpatient and educational settings is vital to changing this disease population's dynamics. New medications are always being developed and testing their efficacy to find better ways to treat COPD.

\section{Limitations}

Data provided for hospitalization and ER visits were specific to metropolitan areas such as Pensacola, Panama City, Panama City Beach, and Tallahassee. Rural county information was not reported in the data set provided for costs. However, metropolitans are more likely to have a pulmonary specialist that monitors patients with COPD. Facilities with specialists can also ascertain a COPD diagnosis, which would have been reported more frequently as a primary cause for admission. The most recent data sets used were reported from 2015. Newer data sets were not accessible at the time of this investigation.

\section{Future Consideration}

Further research is needed to determine costs in rural areas of the Panhandle that do not currently report average costs associated with COPD. Research is needed to determine the specifics for each area, focusing on the following: 
1. Is there missing or not reported data from the rural counties?

2. Are patients in rural areas more compliant with medical treatment? If so, why?

3. How can we improve COPD care in the Panhandle to lower healthcare costs and improve health outcomes?

\section{Acknowledgments}

I would like to express my gratitude to Dr. Jackman for her insight with this write-up.

\section{References}

Agency for Health Care Administration (AHCA). (2017). Condition/Procedures: Chronic Obstructive Pulmonary Disease, COPD [Data file]. Retrieved from http://www.floridahealthfinder.gov/CompareCare/CompareFacilities.aspx?ProdLine=108\&AgeGroup $=3$

American Thoracic Society. (2013). Patient information series. Chronic Obstructive Pulmonary Disease (COPD). Retrieved from https://www.thoracic.org/patients/patient-resources/resources/copd-intro.pdf

Bhattarai, B., Walpola, R., Mey, A., Anoopkumar-Dukie, S., \& Khan, S. (2020). Barriers and Strategies for Improving Medication Adherence Among People Living With COPD: A Systematic Review. Respiratory Care.

Center for Disease Control and Prevention (CDC). (2018). Chronic obstructive pulmonary disease COPD. Retrieved from https://www.cdc.gov/copd/infographics/copd-costs.html

Davis, J. R., Wu, B., Kern, D. M., Tunceli, O., Fox, K. M., Horton, J., Legg, R. F. \& Trudo, F. (2017). Impact of Nonadherence to Inhaled Corticosteroid/LABA Therapy on COPD Exacerbation Rates and Healthcare Costs in a Commercially Insured US Population. American health \& drug benefits, 10(2), 92102 .

Florida Health. (2018). Chronic obstructive pulmonary disease. Florida Environmental Public Health Tracking. Retrieved from https://www.floridatracking.com/healthtracking/mapview.htm?i=5253\&g=3\&t=2017 Garvey, C., \& Kaplan, A. G. (2018). Optimizing Adherence to Improve Clinical Outcomes in Patients with Chronic Obstructive Pulmonary Disease. Journal of Family Practice, 67(10), S40-S40.

Guarascio, A. J., Ray, S. M., Finch, C. K., \& Self, T. H. (2013). The clinical and economic burden of chronic obstructive pulmonary disease in the USA. ClinicoEconomics and outcomes research: CEOR, 5, $235-245$. doi:10.2147/CEOR.S34321

Patel, J. G., Coutinho, A. D., Lunacsek, O. E., \& Dalal, A. A. (2018). COPD affects worker productivity and health care costs. International journal of chronic obstructive pulmonary disease, 13, 2301-2311. doi:10.2147/COPD.S163795

Restrepo, R. D., Alvarez, M. T., Wittnebel, L. D., Sorenson, H., Wettstein, R., Vines, D. L., Sikkema-Ortiz, J., Gardner, D. D., \& Wilkins, R. L. (2008). Medication adherence issues in patients treated for COPD. International journal of chronic obstructive pulmonary disease, 3(3), 371-84. 
Sethi, S. (2018). Effective management of COPD in primary care: Challenges and opportunities. AJMC. Retrieved from https://www.ajmc.com/contributor/sanjay-sethi/2018/11/effective-management-of-copd-in-primarycare-challenges-and-opportunities

Smith, A. L., Palmer, V., Farhat, N., Kalus, S., Thavarajah, K., DiGiovine, B., MacDonald, N. (2017). HospitalBased Clinical Pharmacy Services to Improve Ambulatory Management of Chronic Obstructive Pulmonary Disease. Journal of Pharmacy Technology. 33(1), 8-14. Retrieved from http://journals.sagepub.com/doi/10.1177/8755122516675635 